\title{
NIVEL DE INFECCIÓN DEL MICROSPORIDIO Nosema Spp. EN COLMENAS DE ABEJAS AFRICANIZADAS Y SU RELACIÓN CON LA PRECIPITACIÓN Y HUMEDAD RELATIVA
}

\author{
Rafael A. Calderón-Fallas ${ }^{1 / *}$, Eduardo Moreno-Morales ${ }^{2}$ \\ Palabras clave: Apicultura; Nosemosis; esporas; nivel de infección; época lluviosa. \\ Keywords: Apiculture; Nosemosis; spores; infection level; rainy season.
}

Recibido: 02/12/2020 Aceptado: 14/06/2021

RESUMEN

Introducción. La Nosemosis es una enfermedad de las abejas melíferas, que se encuentra diseminada por todo el mundo y ocasiona pérdidas económicas. Es causada por 2 especies de hongos, Nosema apis y Nosema ceranae. Objetivo. Se determinó la cantidad de esporas de Nosema spp. en muestras grupales e individuales de abejas, y se relacionaron con la precipitación y humedad. Materiales y métodos. El estudio se realizó de julio a noviembre 2017, en 10 colmenas de abejas africanizadas en Atenas, Alajuela. De cada colmena, se colectaron 100 abejas, para examinarlas en el laboratorio de Patología Apícola-CINAT. Para el análisis grupal, se cortaron los abdómenes de 30 abejas y se maceraron con $30 \mathrm{ml}$ de agua destilada. La muestra se revisó en el microscopio a 40x y se cuantificaron las esporas de Nosema spp. mediante el hemocitómetro. Para el examen individual, se utilizó la misma técnica. La precipitación y humedad

\footnotetext{
* Autor para correspondencia. Correo electrónico: rafael.calderon.fallas@una.cr

1 Universidad Nacional, Centro de Investigaciones Apícolas Tropicales, Programa Integrado de Patología Apícola, Heredia, Costa Rica. (D) 0000-0002-6991-6899.
}

\section{ABSTRACT}

Infection level of microsporidium Nosema spp. in africanized honey bee colonies and their relationship with precipitation and relative humidity. Introduction. Nosemosis is the most widespread of adult honey bee diseases that cause significant economic losses worlwide. Honey bees are parasitized by 2 fungus species, Nosema apis and Nosema ceranae. Objective. The amount of spores of Nosema spp. in group and individual samples of adult bees was related with precipitation and relative humidity conditions. Materials and methods. This study was conducted from July to November 2017, in 10 africanized honey bees colonies in Atenas, Alajuela. A 100 foragers bees were collected per colony to examine them at the Bee Pathology Lab of CINAT. For the group analysis, the abdomens of thirty adult bees were cut and macerated together in $30 \mathrm{ml}$ of distilled water. Nosema spp. spores were identified 
relativa durante el estudio, se relacionaron con el número de esporas. Resultados y conclusión. Se detectó el microsporidio Nosema spp. en todas las colmenas analizadas. En julio, se cuantificó el mayor número de esporas, con un promedio de $20360000 \pm 1586957$ en el análisis grupal y 12 $749733 \pm 867232$ en el individual. En los meses siguientes se observó un descenso en el conteo de esporas en ambos métodos, hasta contabilizar la menor cantidad en noviembre, con un promedio de $4375000 \pm 874132$ y $2087708 \pm 398895$ en el examen grupal e individual, respectivamente. Al relacionar la precipitación y humedad con la cantidad de esporas, setiembre presentó la mayor cantidad de lluvia y un alto porcentaje de humedad, sin embargo, no correspondió con el mayor nivel de Nosemosis, el cual se registró en julio. Mientras que noviembre fue el menos lluvioso y húmedo, y mostró la menor cantidad de esporas.

\section{INTRODUCCIÓN}

La alta eficiencia polinizadora de las abejas melíferas (Apis mellifera L.), ha conllevado a una demanda creciente de este servicio a nivel mundial en diversos cultivos agrícolas, como el melón, la sandía y el almendro, entre otros. Sin embargo, se ha reportado en diferentes países como España, Francia, Suiza y Alemania, pérdidas considerables de colmenas. Asimismo, se ha indicado una severa disminución de colonias en diferentes zonas de los Estados Unidos (Botías et al. 2012, Eiri et al. 2015). Sin conocer con exactitud la causa de la pérdida de colmenas, algunos investigadores lo atribuyen a ciertos factores como el uso excesivo de plaguicidas en using a light microscopy at 40x magnification and counted with the hemocytometer. For the individual analysis, the same method was used. Precipitation and relative humidity conditions during the study period were related with the amount of Nosema spp. spores. Results and conclusion. The microsporidium Nosema spp. was found in all colonies. In July, the highest number of Nosema spores were quantified, with an average of $20360000 \pm 1586957$ per bee in group analysis and $12749733 \pm 867232$ in individual analysis. In the following months a decrease in the number of spores was observed and the lowest amount of spores was recorded in November, with an average of $4375000 \pm 874$ 132 and $2087708 \pm 398895$ of spores in group and individual exam, respectively. When relating the conditions of precipitation and humidity with the amount of spores, September presented the highest amount of rain and a high percentage of humidity, nevertheless, it did not correspond to the highest infection level of Nosema spp., which occurred in July. Meanwhile, November was the least rainy and humid month and in turn presented the least amount of spores.

la agricultura y a la presencia de enfermedades en las abejas melíferas, como la Nosemiasis (Soroker et al. 2011, Botías et al. 2012, Tozkar et al. 2015).

La Nosemiasis o Nosemosis, es una enfermedad diseminada por todo el mundo que causa pérdidas económicas en la apicultura (Fries et al. 1996, Fries 2010). En Costa Rica, la Nosemosis es causada por $N$. ceranae, se determinó por primera vez en el 2006 (Calderón et al. 2008). Es ocasionada por 2 especies de microsporidios, Nosema apis y Nosema ceranae, ambas forman esporas y al ser ingeridas por la abeja llegan al intestino, donde infectan las células epiteliales, se desarrollan y multiplican, luego pasan al recto 
y son liberadas con las heces (Traver y Fell 2011, Muñoz et al. 2014).

La Nosemosis se transmite entre las abejas a través del intercambio de alimento (trofalaxis), durante la limpieza de panales por parte de las obreras o mediante la ingestión de esporas presentes en el agua y/o alimento contaminado (Higes et al. 2007, Chihu et al. 2013). La abeja reina se infecta a través de la jalea real proporcionada por las abejas nodrizas enfermas, mientras que los zánganos se contagian al recibir de las obreras, alimento con esporas (Maside et al. 2015). Entre colmenas, la Nosemosis se transfiere por pillaje, deriva, manejo inadecuado por parte del apicultor, o por la introducción de colmenas enfermas a los apiarios (Calderón y Pichardo 2011, Prendas et al. 2018).

La intensidad de la infección varía con la época del año, las condiciones ambientales (temperatura, humedad relativa y precipitación) y la ubicación del apiario (sombra o semisombra) (Fries 2010, Traver y Fell 2011, Jack et al. 2016, Ptaszynska et al. 2016). Se ha reportado, que apiarios ubicados en lugares húmedos y con exceso de sombra, son propensos a presentar niveles elevados de Nosema spp. (Biganski et al. 2017). La Nosemosis puede permanecer latente durante todo el año y manifestarse después de periodos de hacinamiento de las abejas dentro de la colmena, debido a lluvias persistentes, fríos intensos o fuertes vientos, ya que estas condiciones favorecen el contacto y contagio entre las abejas (Calderón y Ramírez 2013). Algunos de los principales síntomas que se observan en abejas, A. mellifera, altamente infectadas por Nosema spp., son abejas arrastrándose en frente de la colmena debido a la imposibilidad de volar por la compresión de los sacos aéreos abdominales, así como deyecciones diarreicas de color café sobre los marcos, bastidores y frente a la piquera (Chihu et al. 2013). Lo anterior causa disminución en la población de abejas adultas y en la cantidad de cría, reducción en la producción de miel y en casos muy severos, perdida de la colmena (Chihu et al. 2013).
El diagnóstico de laboratorio de la Nosemosis, consiste en el análisis de una muestra grupal de aproximadamente 30 a 60 abejas adultas por colmena, mediante el cual se obtiene un estimado de la cantidad de esporas por abeja (Calderón y Sánchez 2011, Botías et al. 2012). Sin embargo, se ha reportado que, al aplicar esta metodología, en condiciones particulares, podrían obtenerse resultados muy variables en la medición de infección entre individuos de una misma colmena (Mulholland et al. 2012). Algunas personas autoras mencionan que el análisis individual de abejas es un indicador más confiable del nivel de infección de la colonia. No obstante, estudios sobre la presencia de Nosema spp. en abejas individuales, son escasos (Higes et al. 2008, Mulholland et al. 2012).

Debido a la poca investigación relacionada con la Nosemosis en abejas africanizadas bajo condiciones tropicales, se realizó el presente estudio con el objetivo de determinar la cantidad de esporas y el nivel de infección del microsporidio Nosema spp. en muestras grupales e individuales de abejas adultas y su relación con las condiciones de precipitación y humedad relativa.

\section{MATERIALES Y MÉTODOS}

Ubicación del apiario. La investigación se realizó en un apiario constituido por 10 colmenas de abejas africanizadas (A. mellifera), ubicado en el cantón de Atenas (09 $57^{\circ} 45^{\prime \prime} \mathrm{N}$ y $84^{\circ} 21^{\prime} 50^{\prime \prime}$ O, $535 \mathrm{msnm}$ ), provincia de Alajuela. Atenas se clasifica como bosque húmedo premontano, con una temperatura promedio anual de $25^{\circ} \mathrm{C}$ y una precipitación entre 1011 y 2022 mm (IMN 2017). El estudio se llevó a cabo de julio a noviembre 2017 (época lluviosa), meses en los cuales se ha reportado una mayor incidencia de Nosemosis en las colmenas (Calderón y Pichardo 2011, Pichardo et al. 2012, Calderón y Ramírez 2013). Se confirmó la presencia de Nosema spp. mediante análisis de laboratorio de las abejas adultas, las cuales adquirieron el microsporidio de manera natural. 
Colecta de abejas. Para realizar el diagnóstico de Nosemosis, se muestrearon 10 colmenas con un intervalo de 30 días entre los muestreos. De cada colmena se colectaron aproximadamente 100 abejas adultas de la piquera (entrada), con el fin de muestrear abejas pecoreadoras (con más de 21 días de edad) (Higes et al. 2010, OIE 2013, Medina et al. 2014). Para obtener la muestra de abejas, la piquera de la colmena se cerró temporalmente con papel periódico, durante unos 20 - 30 minutos en horas de la mañana (9:00 am - 11:00 am) (Higes et al. 2008). $\mathrm{Al}$ observarse un número considerable de abejas pecoreadoras agrupadas frente a la piquera, se procedió a colectarlas en un frasco de boca ancha con alcohol etílico al 70\%. Las muestras se identificaron con la fecha y el número de la colmena.

Análisis de abejas. Las abejas adultas se analizaron en el Laboratorio de Patología Apícola del Centro de Investigaciones Apícolas Tropicales (CINAT), Universidad Nacional, ubicado en el Campus Benjamín Núñez, Lagunilla de Heredia. Para determinar la presencia de esporas de Nosema spp., las abejas se analizaron mediante el método de Cantwell (método directo) (Molina et al. 1990, Calderón y Sánchez 2007). De cada muestra se tomaron 30 abejas adultas, se procedió a cortar los abdómenes, los cuales se maceraron en un mortero y se añadió $1 \mathrm{ml}$ de agua destilada por cada abdomen (total $30 \mathrm{ml}$ ). El macerado se agitó constantemente durante un minuto y luego se colocó una gota de la suspensión en un portaobjetos. Para identificar la presencia del microsporidio, la muestra se revisó en el microscopio a un aumento de 40x. Las esporas se identificaron por ser ovaladas, brillantes y refringentes.

\section{Cuantificación del nivel de infección de}

Nosema spp. Se procedió a cuantificar las esporas y determinar el nivel de infección de cada colmena mediante el hemocitómetro (Calderón y Pichardo 2011). Se realizó el análisis grupal de 30 abejas y el examen individual de 30 abejas provenientes de la misma colmena.
Evaluación de una muestra grupal de abejas. Para cuantificar las esporas de Nosema spp. presentes en una muestra grupal de abejas, se procedió a agitar de manera mecánica el líquido sobrenadante para homogenizarlo y con una micropipeta se tomó una alícuota de $200 \mu \mathrm{l}$, la cual se colocó en el hemocitómetro y se dejó reposar durante 3 minutos, para permitir la sedimentación de las esporas. La muestra se observó en el microscopio a un aumento de 40x y se procedió al conteo de esporas de 5 bloques del hemocitómetro (los 4 de las esquinas y el central) (Molina et al. 1990).

El nivel de infección se determinó con la siguiente fórmula:

Número de esporas por abeja $=($ total $\mathrm{de}$ esporas contadas / 80) x 4000000 .

El total de esporas contadas, son las presentes en los 5 bloques del hemocitómetro.

El 80, representa el total de campos contados en los 5 bloques del hemocitómetro.

Los 4000 000, corresponde al volumen de dilución de la muestra.

Examen de abejas individuales. Para analizar el nivel de infección de Nosema spp. en 30 abejas por colmena ( $\mathrm{n}=10$ colmenas), igualmente se utilizó la técnica del hemocitómetro. Se analizó cada una de las abejas de manera individual $(\mathrm{n}=$ 300 abejas analizadas por mes), con el macerado del abdomen y se agregó $1 \mathrm{ml}$ de agua destilada. Luego se colocó $10 \mu$ l del líquido sobrenadante en el hemocitómetro, se observó en el microscopio a un aumento de 40x y se procedió al conteo de las esporas. Para obtener el nivel de infección se utilizó la fórmula indicada anteriormente.

El procedimiento se repitió hasta determinar el nivel de infección de cada una de las 30 abejas analizadas individualmente por colmena.

Nivel de infección de Nosemosis. Para categorizar el nivel de infección de Nosema spp., el número de esporas de cada muestra, determinado mediante la fórmula, se comparó con la tabla de Jaycox (Tabla 1). 
Tabla 1. Nivel de intensidad de infección por Nosemosis en abejas melíferas (Jaycox).

\begin{tabular}{lcc}
\hline Nivel de infección & Intensidad & Cantidad de esporas \\
\hline Nulo & 0 & Menos de 10000 \\
Muy leve & 1 & $10000-1000000$ \\
Leve & 2 & $1000001-5000000$ \\
Moderado & 3 & $5000001-10000000$ \\
Semifuerte & 4 & $10000001-20000000$ \\
Fuerte & 5 & Superior a 20000000 \\
\hline
\end{tabular}

Molina et al. 1990.

Datos meteorológicos. Los datos de precipitación, humedad relativa y temperatura en el cantón de Atenas durante julio a noviembre, se proporcionaron por el Instituto Meteorológico Nacional (IMN 2017). La precipitación y humedad se relacionaron con el número de esporas del microsporidio Nosema spp. en muestras grupales e individuales de abejas adultas.

Análisis estadístico. La cantidad de esporas, obtenida en el análisis de muestras grupales, se digitó en una tabla de Microsoft Excel ${ }^{\circledR}$, la cual se ingresó al programa estadístico Minitab ${ }^{\circledR}$, siendo clasificadas por el número de colmena y el mes de evaluación. Asimismo, se digitó el número de esporas determinadas en muestras de abejas individuales, se incluyeron como variables el número de abeja, el número de colmena y el mes de evaluación. En este último caso, se obtuvo el promedio de esporas presentes en abejas individuales por colmena. Para la cantidad de esporas, se calcularon estadísticas descriptivas (promedio e intervalos de confianza, la mediana, el valor máximo y mínimo) y medidas de variación (desviación estándar, error estándar y el coeficiente de variación) por mes y para cada método de evaluación. La dinámica de la infección de Nosema spp. durante el estudio, se presentó en gráficos de líneas, en los que se utilizó el promedio de esporas de abejas individuales por colmena. La precipitación y humedad relativa se relacionaron con el número de esporas de Nosema spp., determinadas mediante los métodos grupal e individual.

\section{RESULTADOS}

\section{Nosemosis en muestras grupales de abejas}

Se determinó la presencia del microsporidio Nosema spp. en todas las colmenas analizadas $(n=10)$, durante la época lluviosa. En julio, se determinó la mayor cantidad de esporas de Nosema spp. en el apiario, con un promedio de $20360000 \pm 1586957$ por abeja. En el mes siguiente la infección fue significativamente inferior, encontrándose en promedio $9595000 \pm$ 2602866 esporas $(\mathrm{p}<0,0001)$. En setiembre, se cuantificaron $6230000 \pm 1396945$ de esporas por abeja, mientras que en octubre se registró un leve incremento. Finalmente, en noviembre, se obtuvo la menor cantidad de esporas, con un promedio de $4375000 \pm 874132$ (Figura 1). No se encontraron diferencias significativas de agosto a noviembre (ANOVA - significancia 0,05) (Figura 2). 


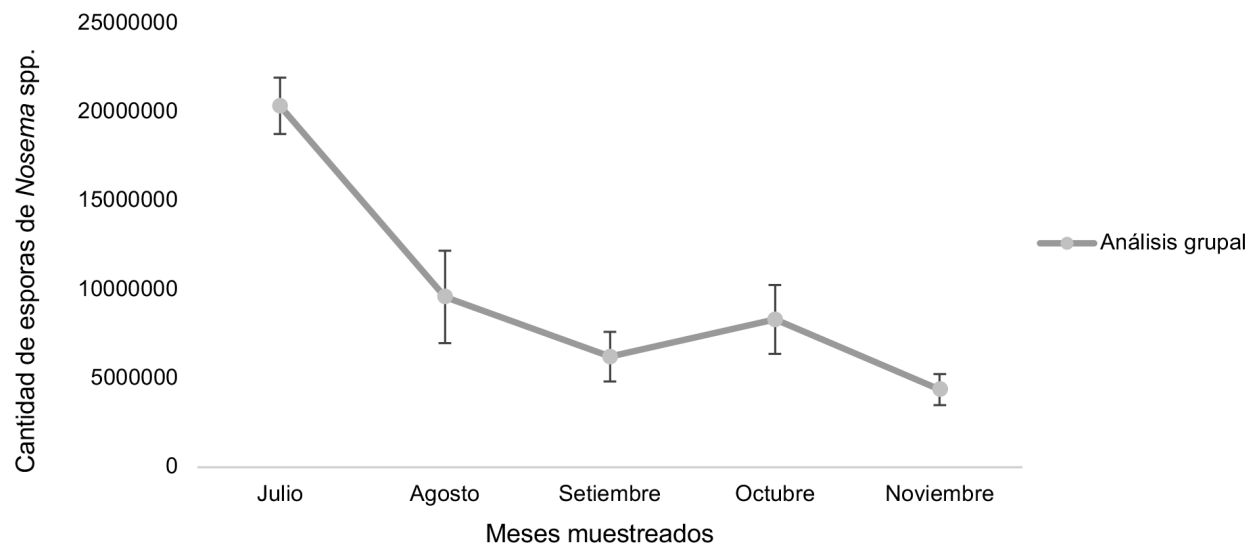

Figura 1. Esporas de Nosema spp. en muestras grupales de abejas adultas durante la época lluviosa $(\overline{\mathrm{x}} \pm \mathrm{SE})$.

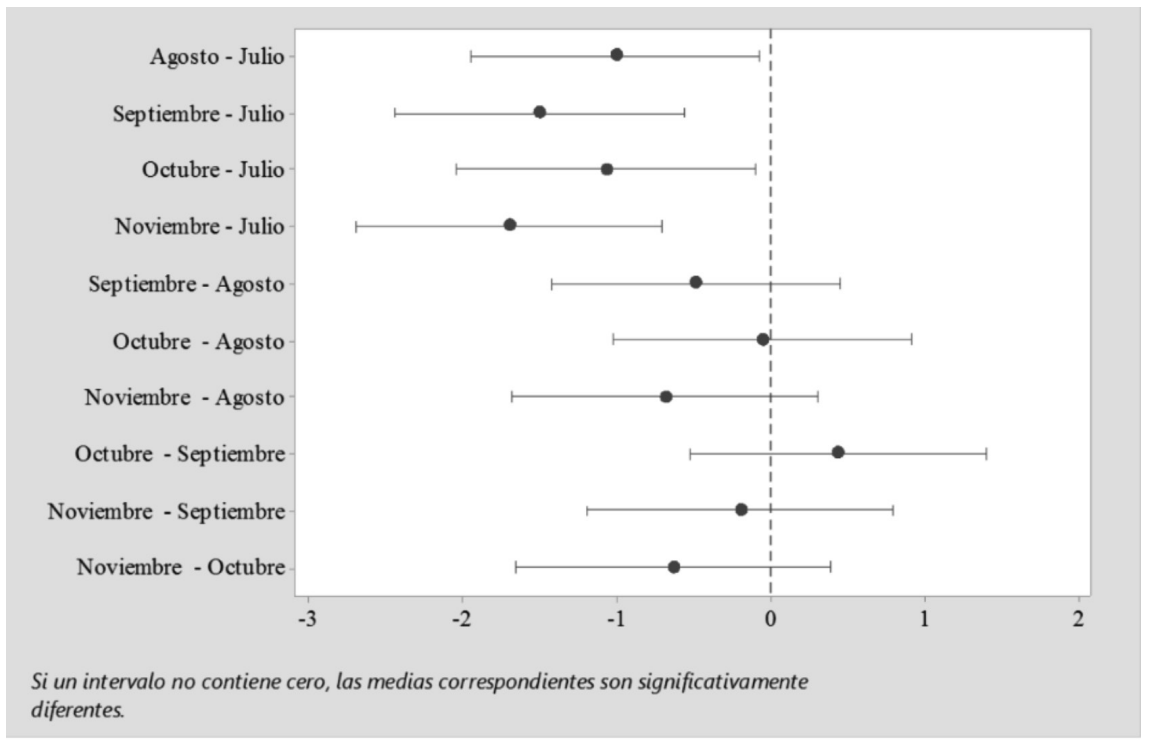

Figura 2. Análisis de la cantidad de esporas de Nosema spp. durante los meses muestreados (ANOVA= Prueba de Tukey, significancia 0,05$)$.

\section{Nosemosis en muestras individuales de abejas}

Se determinó la presencia del microsporidio Nosema spp. en muestras de abejas individuales ( 240 a 300 abejas por mes) durante el periodo de estudio. En julio se contabilizó la mayor cantidad de esporas, con un promedio de $12749733 \pm 867232$ por abeja, y resultó significativamente mayor que los siguientes meses analizados, en los cuales la infección por Nosemiasis fue menor a 10000000 de esporas. En agosto se cuantificó la segunda cifra más alta de esporas, siendo de $7367267 \pm 716697$ por abeja. Durante setiembre y octubre el promedio continuó con disminución, hasta noviembre donde se alcanzó la menor cantidad $2087708 \pm 398895$ de esporas por abeja (Figura 3). 


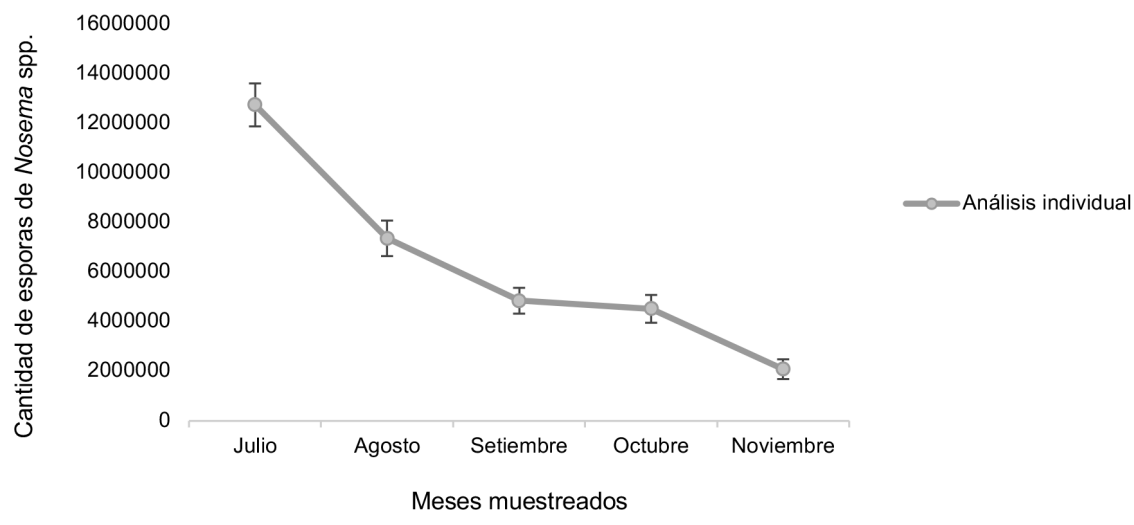

Figura 3. Promedio del conteo de esporas de Nosema spp. en muestras individuales de abejas durante los meses de la época lluviosa $(\overline{\mathrm{x}} \pm \mathrm{SE})$.

Relación de las condiciones de precipitación y humedad relativa con la cantidad de esporas de Nosema spp.

De acuerdo con la información suministrada por el Instituto Meteorológico Nacional (IMN), las variables meteorológicas que presentaron cambios en los meses de estudio, son la precipitación y la humedad relativa, mientras que la temperatura se mantuvo constante entre $23,9^{\circ} \mathrm{C}$ y $24,2^{\circ} \mathrm{C}$. Al relacionar la precipitación y el porcentaje de humedad relativa con la cantidad de esporas, tanto en el análisis grupal como en el individual, se observa que setiembre presentó la mayor cantidad de lluvia $(650 \mathrm{~mm})$ y un alto porcentaje de humedad (90,4\%); sin embargo, no correspondió con los mayores niveles de infección del microsporidio, los cuales se observaron en julio. Mientras que noviembre, fue el mes menos lluvioso (126 mm) y húmedo (87,5\%), y a su vez presentó la menor cantidad de esporas por abeja (Figuras 4 y 5).

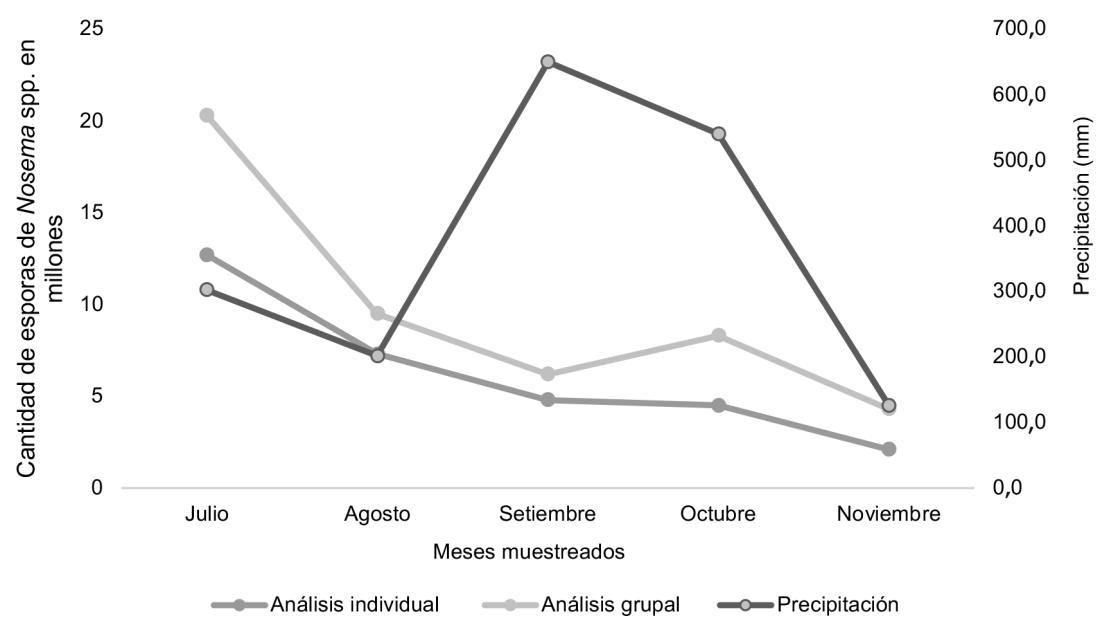

Figura 4. Esporas de Nosema spp. obtenidas en muestras grupales e individuales de abejas y nivel de precipitación entre julio y noviembre. 


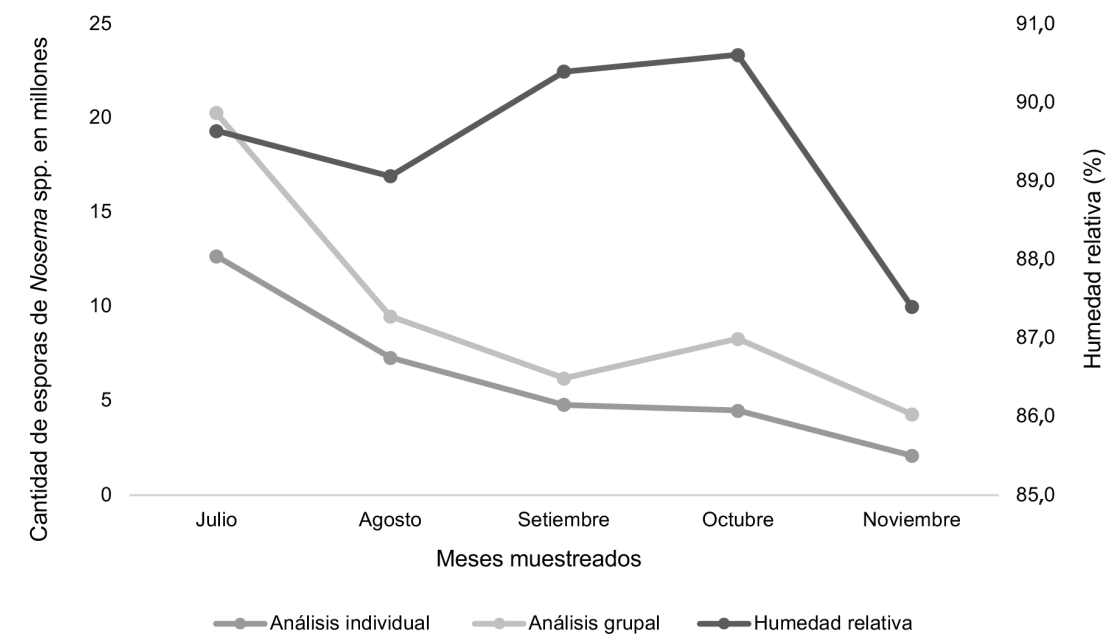

Figura 5. Conteo de esporas de Nosema spp. en muestras grupales e individuales de abejas y porcentaje de humedad relativa durante el periodo de estudio.

\section{DISCUSIÓN}

Como se mencionó, la Nosemosis es una de las enfermedades que más pérdidas económicas causa en la apicultura mundial (Fries et al. 2013, Grupe y Quandt 2020). Diversos estudios en España, indican un aumento considerable en la pérdida de colmenas de abejas de tipo europeo infectadas con $N$. ceranae (Higes et al. 2010, Botías et al. 2013). En esta investigación con abejas africanizadas, se determinó la presencia de Nosemosis en todas las colmenas (prevalencia $=100 \%$ ), tanto en el análisis grupal como en el individual, con diferentes niveles de infección, desde muy leves hasta fuertes. Algunos factores que favorecen a la Nosemosis están relacionados con la ingesta de esporas. Se menciona que las abejas jóvenes adquieren las esporas cuando realizan actividades de limpieza en colmenas contaminadas (Martínez et al. 2011). Según Ritter (2001) panales viejos en la cámara de cría, propician la presencia de Nosemosis. En el presente ensayo, se observaron panales muy oscuros en las colmenas, con mucho tiempo de no cambiarse. En estas condiciones, podrían ser una fuente de contaminación de Nosema spp., ya que las esporas suelen permanecer viables, hasta por un año (DeGrandi-Hoffman y Chen 2015, Rodríguez et al. 2021).

El mayor conteo de esporas obtenido en ambos métodos durante la época lluviosa se registró en julio. Una de las posibles causas podría estar relacionada con el hacinamiento de las abejas debido a lluvias prolongadas. Aun en julio que no fue el mes con mayor precipitación (302 mm), ocasionalmente se presentaron lluvias persistentes desde horas de la mañana, y permanecieron durante todo el día. Debido a que las abejas en estas condiciones no pueden defecar fuera de la colmena y al estrecho contacto entre ellas, probablemente favoreció la diseminación del microsporidio. Se ha reportado que largos periodos de encierro de las abejas en la colmena (hacinamiento), ocasionados principalmente por lluvias persistentes, promueven la presencia de Nosema spp. Entre más extensa es la duración del hacinamiento, mayor es la cantidad de esporas, ya que los niveles de infección se elevan considerablemente, debido al contacto estrecho entre las abejas (Fries et al. 2013).

En los siguientes meses (agosto - octubre), hubo un descenso en la cantidad de esporas con respecto a julio, tanto en el análisis grupal como 
en el individual. Lo anterior representa un cambio considerable respecto a lo esperado, ya que las condiciones de precipitación y humedad, que se han reportado, favorecen el incremento de la Nosemosis, en setiembre $(650 \mathrm{~mm}, 90,4 \%)$ y octubre (540 mm, 90,6\%) (IMN 2017). Las causas de esta disminución, no se conocen con exactitud, no obstante, se debe mencionar que durante esos meses se presentaron las condiciones propias de la época lluviosa, con mañanas soleadas y tardes lluviosas. Esta condición, permitió que las abejas realizaran sus actividades fuera de la colmena durante horas tempranas del día, sobre todo la defecación. Estos resultados coinciden con los reportados por Tapia et al. (2017) con abejas africanizadas en México, donde determinaron una disminución en el conteo de esporas durante la estación lluviosa, comparado con la época seca. Igualmente, Emsen et al. (2015) indicaron bajos niveles de infección de Nosema spp. durante el invierno, los cuales aumentaron en el verano. Adicionalmente, Retschnig et al. (2017) indican que la mayor intensidad del microsporidio se presentó en invierno.

En noviembre, se registró con ambos métodos, el conteo más bajo de esporas. Se debe señalar que, durante este periodo, se observó una reducción en la cantidad de lluvia, con el inicio de la transición hacia la época seca, con las condiciones más bajas de precipitación (126,2 $\mathrm{mm}$ ) y humedad (87,4\%) (IMN 2017). Lo anterior coincide con Calderón y Ramírez (2013), quienes indican que a menor cantidad de lluvia las esporas de Nosema spp. disminuyen.

\section{Precipitación y humedad relativa}

Al relacionar el número de esporas del microsporidio Nosema spp. (obtenido en ambos métodos) con la precipitación y humedad, se evidenció durante julio la cifra más elevada de esporas en el apiario, mientras que la humedad y precipitación correspondieron a la tercera más alta registrada durante el periodo de investigación. Como se indicó, el conteo elevado de esporas en julio, podría relacionarse con el hacinamiento de las abejas como consecuencia de lluvias persistentes.

Cueto et al. (2020) indicaron una alta prevalencia y distribución de Nosemosis entre marzo y junio, lo cual corresponde a las temporadas de primavera e inicio del verano (mayor actividad estacional de las abejas melíferas) en 4 regiones geográficas del noroeste de México.

Setiembre, presentó la mayor cantidad de lluvia y un alto porcentaje de humedad, no obstante, no correspondió con los mayores niveles de infección de Nosema spp. en las colmenas. Según Rangel et al. (2015) esto obedece a que la lluvia, no es un factor que determina el nivel de infección de Nosemosis en el apiario. Pacini et al. (2016) indican que apiarios ubicados en distintas eco-regiones de Argentina, mostraron diferencias en la cantidad de esporas, al registrar conteos menores en condiciones de bosque y en lugares con suelo cubierto de pasto. Sobre esta condición Calderón y Ramírez (2013), reportaron que apiarios ubicados en sitios húmedos y con mucha sombra, tienden a presentar niveles más elevados de infección, que los situados en lugares donde predominen las condiciones secas y soleadas.

Se comprobó que en noviembre se cuantificó la menor cantidad de esporas de Nosema spp. por abeja. En ese mes se observó una reducción considerable en la cantidad de lluvia y el inicio de la floración. Lo anterior coincide con lo observado por Calderón y Ramírez (2013), quienes encontraron que, a menor cantidad de lluvia, se reduce el número de esporas en el apiario.

En conclusión, se observó que, al relacionar la precipitación y el porcentaje de humedad relativa con la cantidad de esporas, tanto en el análisis grupal como el individual, el mes de setiembre presentó la mayor cantidad de lluvia y un alto porcentaje de humedad, sin embargo, no correspondió con los mayores niveles de infección de Nosemosis, los cuales se presentaron en julio. Mientras que noviembre, fue el periodo menos lluvioso y húmedo, y a su vez presentó la menor cantidad de esporas de Nosema spp. por abeja. 


\section{AGRADECIMIENTOS}

Deseamos agradecer a los estudiantes que prepararon muestras de abejas adultas en el laboratorio. Un agradecimiento a M.Sc. Fernando Ramírez Arias y Tec. Guillermo Ramírez Arias (CINAT-UNA), por colaborar en el manejo general de las colmenas durante el estudio.

\section{LITERATURA CITADA}

Biganski, S; Kurze, C; Müller, M; Moritz, R. 2017. Social response of healthy honeybees towards Nosema ceranae-infected workers: care or kill? Apidologie 49(3):325-334.

Botías, C; Martín-Hernández, R; Barrios, L; Meana, A; Higes, M. 2013. Nosema spp. infection and its negative effects on honeybees (Apis mellifera iberiensis) at the colony level. Veterinary Research 44:25. DOI: http:// dx.doi.org/10.1186/1297-9716-44-25.

Botías, C; Martín-Hernández, R; Meana, A; Higes, M. 2012. Critical aspects of the Nosema spp. diagnostic sampling in honeybee (Apis mellifera L.) colonies. Parasitology Research 110:2557-2561.

Calderón, RA; Pichardo, J. 2011. Nosemiasis en abejas melíferas: diagnostico, control y prevalencia. Heredia, Costa Rica. Departamento de Publicaciones e Impresiones UNA. 52 p.

Calderón, RA; Ramírez, F. 2013. Enfermedades de las abejas melíferas, con énfasis en abejas africanizadas. Heredia, Costa Rica. Editorial UNA (EUNA). 137 p.

Calderón, RA; Sánchez, L; Yañez, O; Fallas, N. 2008. Presence of Nosema ceranae in Africanized honey bee colonies in Costa Rica. Journal of Apicultural Research and Bee World 47:328-329.

Calderón, RA; Sánchez, L. 2007. Detección de enfermedades en abejas africanizadas en Costa Rica. Revista Ciencias Veterinarias 25(2):335-348.

Calderón, RA; Sánchez, L. 2011. Diagnóstico de enfermedades en colmenas de abejas africanizadas en Costa Rica: prevalencia y distribución de setiembre a noviembre del 2007. Agronomía Costarricense 35(2):49-60.

Chihu, L; Chihu, D; Fernández, M. 2013. Nosema ceranae, un patógeno emergente en la apicultura mundial. Leipzig, Alemania. Editorial Académica española. $112 \mathrm{p}$.

Cueto, SA; López, G; Orozco, C; Gómez, SD; Moreno, K; Espinoza, KO; Guerrero, JG; Silva, LE; Trasviña, E; Monge, FJ. 2020. Prevalence and geographical distribution of Nosema apis and Nosema ceranae in apiaries of Northwest of Mexico using a duplex real time PCR with melting curve. Journal of Apicultural Research 59(2):195-203.
DeGrandi-Hoffman, G; Chen, Y. 2015. Nutrition, immunity and viral infections in honeybees. ScienceDirect 10:170-176.

Eiri, D; Suwannapong, G; Endler, M; Nieh, J. 2015. Nosema ceranae can infect honeybee larvae and reduces subsequent adult longevity. PLOS ONE 10(5):1-17.

Emsen, B; Guzman-Novoa, E; Hamiduzzaman, M; Eccles, L; Lacey, B; Ruiz-Pérez, R; Nasr, M. 2015. Higher prevalence and levels of Nosema ceranae than Nosema apis infections in Canadian honeybee colonies. Parasitology Research 115(1):175-181.

Fries, I; Chauzat, M; Chen, Y; Doublet, V; Genersh, E; Gister, S; Williams, G. 2013. Standard methods for Nosema research. Journal of Apicultural Research 52(1):1-28.

Fries, I; Feng, F; Da Silva, A; Slemenda, SB; Pieniazek, J. 1996. Nosema ceranae (Microspora, Nosematidae), morphological and molecular characterization of a microsporidian parasite of the Asian honeybee Apis ceranae (Hymenoptera, Apidae). Journal of Protistology 32:356-365.

Fries, I. 2010. Nosema ceranae in European honeybees (Apis mellifera). Journal of Invertebrate Pathology 103:S73-S79.

Grupe, AC; Quandt, CA. 2020. A growing pandemic: A review of Nosema parasites in globally distributed domesticated and native bees. PLoS Pathogens 16(6):1-8.

Higes, M; García-Palencia, R; Martín-Hernández, R; Meana, A. 2007. Experimental infection of Apis mellifera honeybees with Nosema ceranae (Microsporidia). Journal of Invertebrate Pathology 94(3):211-217.

Higes, M; Martín-Hernández, R; Botías, C; Bailón, EG; González-Porto, AV; Barrios, L; Meana, A. 2008. How natural infection by Nosema ceranae causes honeybee colony collapse. Environmental Microbiology 10(10):2659-2669.

Higes, M; Martin-Hernández, R; Meana, A. 2010. Nosema ceranae in Europe: an emergent type C Nosemosis. Apidologie 41:375-392.

IMN (Instituto Meteorológico Nacional). 2017. Regiones y subregiones climáticas del país (en línea). Consultado 14 may. 2018. Disponible en https://www.imn.ac.cr/ publicaciones/estudios/Reg_climaCR.pdf

Jack, C; Lucas, H; Webster, T; Sagili, R. 2016. Colony level prevalence and intensity of Nosema ceranae in honeybees (Apis mellifera L.). PLOS ONE 11(9):1-20.

Martínez, J; Medina, L; Catzín, A. 2011. Frecuencia de Varroa destructor, Nosema apis y Acarapis woodi en colonias manejadas y enjambres silvestres de abejas (Apis mellifera) en Mérida, Yucatán, México. Revista Mexicana de Ciencias Pecuarias 2(1):25-38.

Maside, X; Gómez-Moracho, T; Jara, L; Martín-Hernández, R; De la Rúa, P; Higes, M; Bartolomé, C. 2015. Population genetics of Nosema apis and Nosema 
ceranae: One host (Apis mellifera) and two different histories. PLOS ONE 10(12):1-21.

Medina, C; Guzmán, E; Espinosa, L; Uribe, L; Gutiérrez, R; Gutiérrez, F. 2014. Frecuencia de Varroosis y Nosemosis en colonias de abejas melíferas (Apis mellifera) en el estado de Zacatecas, México. Revista Chapingo serie Ciencias Forestales y del Ambiente 10:30-36.

Molina, A; Guzmán, E; Message, D; De Jong, D; Pesante, D; Mantilla, C; Meneses, G. 1990. Enfermedades y plagas de la abeja melífera occidental. San Salvador, El Salvador. OIRSA-BID. 147 p.

Mulholland, G; Traver, B; Johnson, N; Fell, R. 2012. Individual variability of Nosema ceranae infections in Apis mellifera colonies. Insects 3:1143-1155.

Muñoz, I; Cepero, A; Pinto, M; Martín-Hernández, R; Higes, M; De la Rúa, P. 2014. Presence of Nosema ceranae associated with honeybee queen introductions. Infection, Genetics and Evolution 23:161-168.

OIE (Organización Mundial de Sanidad Animal). 2013. Manual de las pruebas de diagnóstico y las vacunas para los animales terrestres (en línea). Consultado 20 oct. 2019. Disponible en http://www.oie.int/en/ international-standard-setting/terrestrial-manual/ access-online/

Pacini, A; Giaocobino, A; Molineri, A; Bulacio, N; Aignasse, A; Zago, L; Signorini, M. 2016. Risk factors associated whith the abundance of Nosema spp. in apiaries located in temperate and subtropical conditions after honey harvest. Journal of Apicultural Research 55(4):342-350.

Pichardo, J; Calderón, RA; van Veen, J; Ramírez, F. 2012. Nosemiasis en abejas Africanizadas: detección en cámara de cría y piquera. Notas Apícolas 15:26-33.

Prendas-Rojas, J; Figueroa-Mata, G; Ramírez-Montero, M; Calderón-Fallas, RA; Ramírez-Bogantes, M; Travieso-González, C. 2018. Diagnóstico automático de infestación por Nosemiasis en abejas melíferas mediante procesado de imágenes. Revista Tecnología en Marcha 31(2):14-25.

Ptaszynska, A; Paleolog, J; Borsuk, G. 2016. Nosema ceranae infection promotes proliferation of yeasts in honeybee intestines. PLOS ONE 11(10):1-15.

Rangel, J; Baum, K; Rubink, W; Coulson, R; Johnston, J; Traver, E. 2015. Prevalence of Nosema species in a feral honeybee population: a 20 -year survey. Apidologie 47:561-571.

Retschnig, G; Williams, G; Schneeberger, A; Neumann, P. 2017. Cold ambient temperature promotes Nosema spp. intensity in honey bees (Apis mellifera). Insects 8(1):20. DOI: https://dx.doi. org $/ 10.3390 \% 2$ Finsects 8010020 .

Ritter, W. 2001. Enfermedades de las abejas. Zaragoza, España, Editorial Acribia S.A. 146 p.

Rodríguez, C;Higes, M; Martin, R.2021. Sporicidalevaluation of different substances against Nosema ceranae for surface disinfection. Journal of Apicultural Research. DOI: 10.1080/00218839.2021.1933750. Early Access.

Soroker, VA; Hetzroni, B; Yakobson, D; David, A; David, H; Voet, Y. 2011. Evaluation of colony losses in Israel in relation to the incidence of pathogens and pests. Apidologie 42(2):192-199.

Tapia, J; Alcazar, G; Macías, J; Contreras, F; Tapia, J; Chavoya, F; Martínez, J. 2017. Nosemosis en abejas melíferas y su relación con factores ambientales en Jalisco, México. Revista Mexicana de Ciencias Pecuarias 8(3):325-330.

Tozkar, C; Kence, M; Kence, A; Huang, Q; Evans, J. 2015. Metatranscriptomic analyses of honeybee colonies. Frontiers of Genetics 6(100):1-12.

Traver, B; Fell, R. 2011. Prevalence and infection intensity of Nosema in honeybee (Apis mellifera L.) colonies in Virginia. Journal of Invertebrate Pathology 107:43-49. 
